\title{
Presurgical evaluation of pediatric epilepsy patients prior to hemispherotomy: the prognostic value of ${ }^{18}$ F-FDG PET
}

\author{
Tatjana Traub-Weidinger, MD, ${ }^{1}$ Philip Weidinger, MD, ${ }^{2}$ Gundrun Gröppel, MD, ${ }^{2}$ \\ Georgios Karanikas, MD, ${ }^{1}$ Wolfgang Wadsak, PhD, ${ }^{1}$ Gregor Kasprian, MD, ${ }^{1}$ Christian Dorfer, MD, ${ }^{3}$ \\ Anastasia Dressler, MD, ${ }^{2}$ Angelika Muehlebner, MD, ${ }^{2}$ Marcus Hacker, MD, ${ }^{1}$ Thomas Czech, MD, ${ }^{3}$ \\ and Martha Feucht, MD²
}

Departments of ${ }^{1}$ Biomedical Imaging and Image-Guided Therapy, ${ }^{2}$ Pediatrics, and ${ }^{3}$ Neurosurgery, Medical University of Vienna, Austria

OBJECTIVE The objective of this study was to investigate whether fluorine-18 fluorodeoxyglucose PET ( ${ }^{18} \mathrm{~F}-\mathrm{FDG}$ PET) can help to predict seizure outcome after hemispherotomy and therefore may be useful in decision making and patient selection.

METHODS Children and adolescents less than 18 years of age who underwent ${ }^{18} \mathrm{~F}$-FDG PET studies during presurgical evaluation prior to hemispherotomy and had follow-up data of at least 12 months after surgery were included. Seizure outcome was classified according to the recommendations of the International League Against Epilepsy. PET data were reevaluated by two specialists in nuclear medicine blinded to clinical data and to MRI. MRI studies were also reinterpreted visually by an experienced neuroradiologist blinded to clinical data and PET findings.

RESULTS Thirty-five patients ( 17 girls) with a median age of 5 years (range $0.4-17.8$ years) were evaluable. Of the 35 patients, $91.4 \%$ were seizure free after surgery, including $100 \%$ of those with unilateral ${ }^{18} \mathrm{~F}-\mathrm{FDG}$-PET hypometabolism compared with only $75 \%$ of those with bilateral hypometabolism. With respect to MRI, seizure freedom after surgery was observed in $96.4 \%$ of the patients with unilateral lesions compared with only $71.4 \%$ in those with bilateral MRI lesions. The best seizure outcomes were noted in patients with unilateral findings in both PET and MRI (100\% seizure freedom) whereas only $50 \%$ of those with bilateral findings in both imaging techniques were seizure free. Furthermore, $100 \%$ of the patients with unilateral PET hypometabolism and bilateral MRI findings were also seizure free, but only $87.5 \%$ of those with bilateral PET hypometabolism and unilateral MRI findings.

CONCLUSIONS According to these results, candidate selection for hemispherotomy can be optimized by the use of ${ }^{18} \mathrm{~F}-\mathrm{FDG}$ PET as part of a multimodal presurgical evaluation program, especially in patients with inconsistent (bilateral) MRI findings.

http://thejns.org/doi/abs/10.3171/2016.5.PEDS1652

KEY WORDS ${ }^{18}$ F-FDG; PET; hemispherotomy; drug-resistant epilepsy

$\mathrm{H}$ EMISPHERECTOMY has been confirmed to be a highly effective surgical approach for carefully selected children with drug-resistant epilepsies due to hemispheric pathologies., ${ }^{4,711}$ Starting with functional hemispherectomy introduced by Rasmussen, ${ }^{18}$ various techniques have been developed with less resective and more disconnective components described as hemispherotomies. Today, hemispherectomies/hemispherotomies make up approximately $16 \%$ of all epilepsy surgeries performed in pediatric patients. Almost one-third of these surgical candidates are infants younger than 4 years of age.$^{17}$ However, both seizure and developmental outcomes after surgery primarily depend on the functional integrity of the contralateral nonoperated hemisphere.

Presurgical evaluation prior to hemispherotomy is often hampered by inconclusive seizure semiology and interictal/ictal electroencephalography (EEG) as well as bilateral MRI findings. Additional diagnostic tools providing reli-

ABBREVIATIONS ${ }^{18 F-F D G-P E T ~=~ f l u o r i n e-18 ~ f l u o r o d e o x y g l u c o s e ~ P E T ; ~ E E G ~=~ e l e c t r o e n c e p h a l o g r a p h y ; ~ I L A E ~=~ I n t e r n a t i o n a l ~ L e a g u e ~ A g a i n s t ~ E p i l e p s y ; ~ M C D ~=~ m a l f o r m a-~}$ tions of cortical development.

SUBMITTED January 25, 2016. ACCEPTED May 27, 2016.

INCLUDE WHEN CITING Published online September 9, 2016; DOI: 10.3171/2016.5.PEDS1652. 
able lateralizing information to facilitate decision making are therefore needed. Fluorine-18 fluorodeoxyglucose positron emission tomography $\left({ }^{18} \mathrm{~F}\right.$-FDG PET) is an established diagnostic tool that helps to delineate the epileptogenic zone and to gain information about the functional state of the "remaining" parts of the brain, especially in the absence of radiographic lesions. ${ }^{12,17,21}$

In patients with temporal lobe epilepsy, the presence of extratemporal hypometabolism, ipsilateral and/or contralateral to the epileptogenic focus, was associated with higher rates of unfavorable seizure outcomes after surgery. ${ }^{1,2,23,26}$ However, data are limited concerning the prognostic value of PET in children evaluated for hemispherotomy. ${ }^{6,18,19}$ Moosa et al. reported that presurgical ${ }^{18} \mathrm{~F}-\mathrm{FDG}$ PET identifying or excluding hypometabolic areas in the contralateral remaining hemisphere can provide prognostic information. ${ }^{15}$

The purpose of this study was to investigate the lateralizing value of ${ }^{18} \mathrm{~F}-\mathrm{FDG}$ PET compared with MRI in children and adolescents with drug-resistant hemispheric epilepsies. The hypothesis was that PET is a reliable tool to obtain additional information supporting the surgical approach, even in cases with inconclusive or bilateral MRI findings. Therefore, ${ }^{18} \mathrm{~F}-\mathrm{FDG}$ PET and MRI data obtained during the presurgical diagnostic process were reevaluated in relation to seizure outcomes after hemispherotomy.

\section{Methods \\ Study Population}

All clinical data used in this study were obtained from a longitudinal observational database of the pediatric epilepsy center at the Medical University of Vienna maintained by a senior neurologist familiar with the patients (M.F.). The database contains the complete medical records of all children and adolescents $\leq 18$ years of age who have undergone presurgical evaluation and epilepsy surgery since 1999 , including retrospective data on patient demographics, medical history, seizure types, and epilepsy syndromes, as well as prospectively collected data on presurgical evaluation, surgery, histopathology, developmental and seizure outcome, and antiepileptic drug use/ withdrawal after surgery. ${ }^{5,8}$ Follow-up data are collected on an inpatient basis 3 and 12 months after surgery and annually thereafter.

Study inclusion criteria were 1) drug-resistant hemispheric epilepsy; 2) hemispherotomy performed at the study center; 3) availability of 18F-FDG PET studies performed prior to surgery; 4) availability of MRI studies with epilepsy-specific imaging protocols performed prior to surgery; and 5) complete follow-up data of at least 12 months after surgery. This study was approved by the ethics committee of the Medical University of Vienna.

\section{Presurgical Neuroimaging and Interpretation}

${ }^{18} \mathrm{~F}-\mathrm{FDG}$ PET examinations were performed as part of a standardized presurgical evaluation program at the Division of Nuclear Medicine of the Department of Biomedical Imaging and Image-guided Therapy at the Medical University of Vienna. PET acquisition and reconstruction were performed by a dedicated full-ring GE Advance PET scanner (GE Medical Systems). Spatial resolution of the scanner amounts to $4.0 \mathrm{~mm}$ in the axial direction and 3.8 $\mathrm{mm}$ in the tangential direction. ${ }^{18} \mathrm{~F}-\mathrm{FDG}$ was freshly prepared every day according to a well-established method using the GE FASTlab platform (GE Healthcare).${ }^{10}$ After injection of ${ }^{18} \mathrm{~F}-\mathrm{FDG}$ according to the European Association of Nuclear Medicine recommendations for children, a 3-minute transmission scan for attenuation correction was acquired at the beginning of the scanning procedure..$^{25}$ Data acquisition was started 40-60 minutes after injection of ${ }^{18} \mathrm{~F}-\mathrm{FDG}$ and lasted for 15 minutes. Image reconstruction was performed by filtered black projection using a Hanning filter with a cutoff value of $6.2 \mathrm{~mm}$ and a 256 $\times 256$ matrix. Transverse slices of $4.25 \mathrm{~mm}$ were rescaled along the orbitomeatal line and reformatted in coronal and sagittal directions. PET data were retrieved from the internal database of the Division of Nuclear Medicine, and reevaluated with the Hermes Hybrid Viewer (Hermes Medical Solutions) by two specialists in nuclear medicine (T.T.W. and G. Karanikas), who were blinded to the patient's clinical data including video-EEG monitoring results and MRI findings. Brain regions were defined as pathological findings in comparison with the corresponding contralateral area by rating the extent of ${ }^{18} \mathrm{~F}-\mathrm{FDG}$ hypometabolism. In cases of bilateral decrease of ${ }^{18} \mathrm{~F}-\mathrm{FDG}-$ uptake, ipsilateral areas with homogeneous patterns of ${ }^{18} \mathrm{~F}$ FDG detection were used for comparison. Because of the presence of hypometabolism of the cerebellum observed in more than two-thirds of the included patients, this brain region was not used as a reference. The absence of pathological findings was defined as nondecreased ${ }^{18} \mathrm{~F}-\mathrm{FDG}$ uptake in bilateral comparison. The PET results were then classified as unilateral (pathological findings limited to 1 hemisphere), bilateral (pathological findings in both hemispheres), or without any pathological findings.

MRI studies were performed at the Division of Neuro- and Muskuloskeletal Radiology of the Department of Biomedical Imaging and Image-guided Therapy during presurgical evaluation, using either a 1.5-T (Philips Intera) or a 3.0-T machine (Siemens Trio). The protocols applied included at least anatomical, thin-slice volumetric T1-weighted gradient-recalled echo sequences $(1 \mathrm{~mm}$ isotropic resolution), coronal T2-weighted sequences (2-4 $\mathrm{mm}$ slice thickness), axial FLAIR and inversion recovery sequences (2-mm slice thickness), and high-resolution paracoronal FLAIR and T2-weighted sequences acquired perpendicular to the main axis of the hippocampus (2-mm slice thickness). Data were sent to a picture archiving and communication system (Agfa System) and reinterpreted visually by a board-certified neuroradiologist trained in epilepsy and blinded to the patient's clinical data and PET (G. Kasprian).

\section{Seizure Outcome Evaluation After Hemispherotomy}

Seizure outcome after hemispherotomy was classified according to the International League Against Epilepsy (ILAE) proposal based on patient and family reporting. ${ }^{22}$ This classification takes into account fluctuations in seizure frequency after surgery. For this study seizure outcome was simplified to seizure freedom versus ongoing seizures. 
TABLE 1. Demographic and clinical information

\begin{tabular}{|c|c|c|c|c|c|}
\hline Case No. & Age at Surgery (yrs), Sex & Etiology of Epilepsy & Hemispherotomy Side & Follow-Up (mos) & Seizure Outcome* \\
\hline 1 & $3.3, \mathrm{M}$ & MCD & $\mathrm{Lt}$ & 24 & 1a \\
\hline 2 & $0.8, \mathrm{M}$ & Sturge-Weber syndrome & $\mathrm{Rt}$ & 12 & 1a \\
\hline 3 & $17.3, \mathrm{M}$ & Pre-/perinatal stroke & $\mathrm{Lt}$ & 48 & 1a \\
\hline 4 & $5.3, \mathrm{~F}$ & MCD & Rt & 48 & 1a \\
\hline 5 & $8.4, F$ & Pre-/perinatal stroke & Rt & 24 & 1a \\
\hline 6 & $1.8, \mathrm{~F}$ & MCD & Lt & 60 & 1a \\
\hline 7 & $11.7, \mathrm{~F}$ & Pre-/perinatal stroke & Rt & 12 & 1a \\
\hline 8 & $1.4, \mathrm{M}$ & Pre-/perinatal stroke & $\mathrm{Lt}$ & 12 & 1a \\
\hline 9 & $4.7, \mathrm{M}$ & MCD & Rt & 72 & 1a \\
\hline 10 & $6.4, \mathrm{M}$ & Encephalitic & Lt & 12 & 4 \\
\hline 11 & $5.1, M$ & Pre-/perinatal stroke & Rt & 12 & 1a \\
\hline 12 & $0.4, \mathrm{~F}$ & Hemimegalencephaly & Rt & 84 & 1a \\
\hline 13 & $11.0, \mathrm{~F}$ & Tumor-related & Lt & 24 & 1a \\
\hline 14 & $10.8, \mathrm{M}$ & Pre-/perinatal stroke & $\mathrm{Rt}$ & 36 & 1a \\
\hline 15 & $17.7, \mathrm{M}$ & Pre-/perinatal stroke & $\mathrm{Lt}$ & 36 & 1a \\
\hline 16 & $8.7, \mathrm{M}$ & Pre-/perinatal stroke & Rt & 24 & 1a \\
\hline 17 & $20.1, F$ & Pre-/perinatal stroke & Rt & 24 & 1a \\
\hline 18 & $5.5, \mathrm{M}$ & Tumor-related & Lt & 36 & 4 \\
\hline 19 & $0.9, \mathrm{~F}$ & MCD & $\mathrm{Lt}$ & 36 & 1a \\
\hline 20 & $18.8, \mathrm{M}$ & Pre-/perinatal stroke & $\mathrm{Lt}$ & 72 & 1a \\
\hline 21 & $2.1, \mathrm{~F}$ & Pre-/perinatal stroke & Rt & 12 & 1a \\
\hline 22 & $1.0, \mathrm{~F}$ & Post-hemorrhagic & Rt & 12 & 1a \\
\hline 23 & $1.0, \mathrm{M}$ & Sturge-Weber syndrome & Lt & 12 & 1a \\
\hline 24 & $0.7, \mathrm{~F}$ & $M C D$ & Lt & 72 & 4 \\
\hline 25 & $12.3, \mathrm{M}$ & Encephalitic & $\mathrm{Lt}$ & 12 & 1a \\
\hline 26 & $4.9, \mathrm{~F}$ & Pre-/perinatal stroke & $\mathrm{Lt}$ & 48 & 1a \\
\hline 27 & $9.5, M$ & MCD & $\mathrm{Lt}$ & 60 & 1a \\
\hline 28 & $3.5, F$ & Rasmussen encephalitis & Rt & 36 & 1a \\
\hline 29 & $3.5, \mathrm{~F}$ & Pre-/perinatal stroke & $\mathrm{Lt}$ & 48 & 1a \\
\hline 30 & $5.0, \mathrm{~F}$ & Pre-/perinatal stroke & $\mathrm{Lt}$ & 144 & $1 a$ \\
\hline 31 & 3.7, M & Pre-/perinatal stroke & $\mathrm{Lt}$ & 108 & 1a \\
\hline 32 & $1, M$ & Sturge-Weber syndrome & $\mathrm{Lt}$ & 12 & 1a \\
\hline 33 & $6.6, \mathrm{M}$ & MCD & $\mathrm{Lt}$ & 48 & 1a \\
\hline 34 & $2.5, \mathrm{~F}$ & Pre-/perinatal stroke & $\mathrm{Lt}$ & 12 & 1a \\
\hline 35 & $10.3, \mathrm{~F}$ & Pre-/perinatal stroke & $\mathrm{Lt}$ & 36 & 1a \\
\hline
\end{tabular}

* Seizure outcome is classified following the proposal of the 2010 ILAE commission report: 1a = seizure free since surgery; $4=4$ seizure days per year to $50 \%$ reduction of baseline seizure days. ${ }^{6}$

\section{Statistical Analysis}

Because of the small sample size only descriptive analysis was used. Pathological findings and their association with postsurgical outcomes were displayed in absolute and relative frequencies.

\section{Results}

\section{Clinical Data and Seizure Frequencies After Hemispherotomy}

Fifty-five patients were screened, and 20 were excluded from further analysis because of incomplete MRI and/ or PET data. Data from 35 patients (17 girls) with a me- dian age of 5 years at the time of surgery were analyzed. The surgical technique applied in all patients was vertical perithalamic hemispherotomy. All operations were performed by the same neurosurgeon (T. C.) between December 1999 and June 2013.

The underlying pathologies were vascular defects in 18 patients, malformations of cortical development (MCD) in 9, encephalitis including Rassmussen encephalitis in 3 , Sturge-Weber syndrome in 3, and tumors in 2 patients (Table 1). The mean follow-up-period was 39.43 months (range 12-144 months). Seizure freedom (Wieser Class 1a) after surgery was observed in 32 patients $(91.4 \%)$. Three patients $(8.6 \%)$ were not seizure free: Cases 10 , 
19, and 24, who showed seizure outcomes of Wieser Class 4.

\section{${ }^{18}$ F-FDG PET, MRI Findings, and Clinical Outcome After Hemispherotomy}

Because normal ${ }^{18} \mathrm{~F}-\mathrm{FDG}$ distribution patterns in both hemispheres were not found in any of the patients, the study population was divided into two groups with respect to unilateral (Group A, 23/35 patients) or bilateral hypometabolic brain regions (Group B, 12/35 patients). Seizure-freedom after surgery was observed in $100 \%$ (23/23) of the patients in Group A and in 75\% (9/12) of the children in Group B (Table 2).

MRI showed unilateral findings in $79.9 \%$ (28/35) of the children, and $96.4 \%$ (27/28) of them were seizure free after hemispherotomy. MRI was bilateral in $20 \%(7 / 35)$ of the patients, and only $71.4 \%$ (5/7) of them were seizure free after surgery.

According to the results of both imaging modalities, patients were assigned to 4 groups (Groups AI and AII, and Groups BI and BII). As noted in Table 2, seizure freedom after surgery was achieved in $100 \%$ of the patients in Group AII (unilateral PET hypometabolism, bilateral MRI findings) compared with only $87.5 \%$ of the patients in Group BI (bilateral PET hypometabolism, unilateral MRI findings).

\section{Discussion}

At least $35 \%$ of all infants and children with epilepsy are pharmacoresistant. ${ }^{14,24}$ Many of them suffer from early-onset epileptic encephalopathies due to multilobar/ hemispheric pathologies. Hemispheric resection/disconnection has become an increasingly performed and successful treatment option in carefully selected children, and median seizure-free rates of $73.4 \%$ (range 40\%-94\%) have been reported. ${ }^{7}$ In particular, the vertical approach of hemispherotomy was confirmed by our group to be safe and effective, with seizure-free rates of $92 \%{ }^{4,5}$

Prerequisites of favorable outcomes after surgery are the precise lateralization of the hemisphere harboring the epileptogenic zone as well as the proven integrity of the remaining contralateral hemisphere. Unfortunately, many of these infants and young children do not show lateralizing seizure semiology and/or interictal/ictal EEG findings. Neuroimaging studies are therefore of paramount importance during the presurgical evaluation process. Structural MRI gives information about the localization and extent of the epileptogenic lesion and may also reveal structural abnormalities in unaffected brain areas. ${ }^{27}$ However, the significance of contralateral abnormalities in patients evaluated for hemispheric surgery is controversial. Hallbook et al. reported that contralateral MRI findings, less prominent than those in the ipsilateral hemisphere, do not correlate with seizure outcome and therefore may not be a contraindication in otherwise favorable candidates. ${ }^{9}$ In contrast, Moosa et al. observed a poorer outcome in children with bilateral MRI abnormalities after hemispherectomy. ${ }^{16}$

PET-based and SPECT-based techniques have also been investigated with respect to their value for both a
TABLE 2. Presurgical PET and MRI findings in relation to seizure outcomes

\begin{tabular}{cccc}
\hline $\begin{array}{c}\text { Combined PET } \\
\text { \& MRI* }\end{array}$ & $\begin{array}{c}\text { Seizure Free } \\
(\%)\end{array}$ & $\begin{array}{c}\text { Not Seizure Free } \\
(\%)\end{array}$ & Total \\
\hline Group Al & $20100)$ & $0(0)$ & 20 \\
\hline Group All & $3(100)$ & $0(0)$ & 3 \\
\hline Group BI & $7(87.5)$ & $1(12.5)$ & 8 \\
\hline Group BII & $2(50)$ & $2(50)$ & 4 \\
\hline Total & $32(91.4)$ & $3(8.6)$ & 35 \\
\hline
\end{tabular}

* Group AI = unilateral PET hypometabolism, unilateral MRI findings; Group All = unilateral PET hypometabolism, bilateral MRI findings; Group BI = bilateral PET hypometabolism, unilateral MRI findings; Group BII = bilateral PET hypometabolism, bilateral MRI findings. All unilateral abnormalities on PET and MRI were ipsilateral to the operated hemisphere.

better delineation of the functional deficit zone prior to surgery and the prediction of possible functional deficits after surgery. ${ }^{13}$ The largest outcome study after hemispheric surgery (based on 170 patients) was published in $2013 .{ }^{15}$ In this retrospective study, ${ }^{18} \mathrm{~F}-\mathrm{FDG}$ PET as well as MRI findings were found to be of prognostic value for postsurgical seizure outcomes. In addition, multivariate analyses identified bilateral ${ }^{18} \mathrm{~F}$-FDG PET findings as one of the two most valid negative outcome predictors, whereas MRI findings did not correlate with seizure outcome. In our study, we found postoperative seizure freedom in $100 \%$ of the children with unilateral pathological glucose metabolism compared with only $75 \%$ of those with bilateral pathological FDG PET. Our results corroborate those of the Cleveland group (72\% vs 44\% seizure-free rates). Furthermore, seizure freedom after hemispherotomy was observed in $96.4 \%$ of the patients with unilateral versus only $71.4 \%$ of those with bilateral MRI findings. Finally, patients with concordant unilateral findings in both neuroimaging modalities showed seizure free rates of $100 \%$ (Fig. 1). This result underlines the value of a multimodal neuroimaging evaluation prior to hemispherotomy as already recommended by others. ${ }^{12,13}$

Most interestingly, seizure freedom was also achieved in $100 \%$ of the patients with unilateral PET despite bilateral MRI (Group AII; Fig. 2) but only in $87.5 \%$ of the patients with bilateral PET and unilateral MRI (Group BI; Table 2). Our results may reflect the pathophysiological mechanisms of functional deficits, which are suspected to be responsible for hypometabolism in ${ }^{18} \mathrm{~F}-\mathrm{FDG}$ PET. ${ }^{12,20}$ Patients with bilaterally reduced glucose metabolism are suspected to have a "bilateral" disease, even when no structural correlate can be detected by MRI, and thus may have a higher risk of ongoing seizures after surgery.

In summary, PET appears to yield important additional information in patients with unilateral MRI but-almost more importantly-appears to be of crucial importance in cases with bilateral MRI findings. In this context, the radiation exposure by PET imaging as well as the semi-invasive procedure of general anesthesia, which is needed in most of the infants and children with early onset epilepsy syndromes, seems to be justified. 

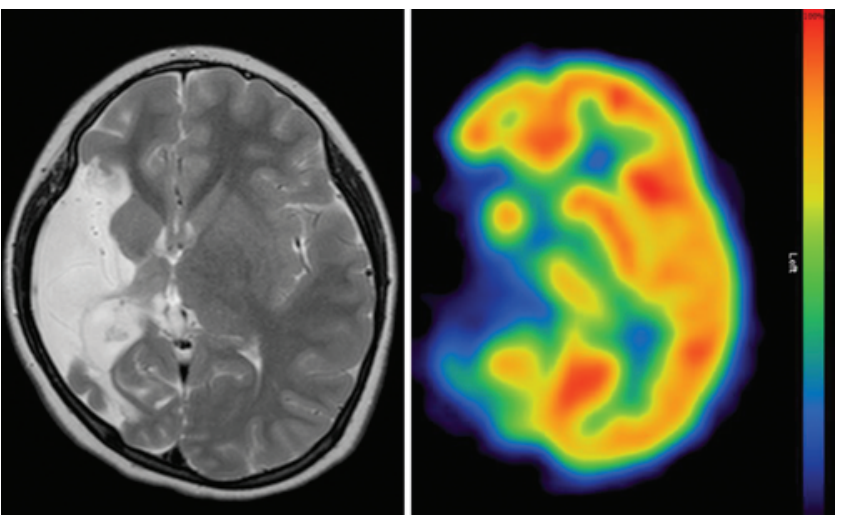

FIG. 1. Case 4. Imaging results from a girl who suffered a perinatal stroke, showing unilateral results in PET as well MRI prior to hemispherotomy. Seizure freedom was observed after surgery over a followup period of 48 months. The axial T2-weighted MRI sequence (left) revealed a large parenchymal defect in the territory of the right middle cerebral artery. In the axial ${ }^{18} \mathrm{~F}$-FDG PET view (right), a large tracer defect corresponding to the parenchymal defect of the right hemisphere is present. Figure is available in color online only.

\section{Limitations of the Study}

The results of ${ }^{18} \mathrm{~F}$-FDG PET have to be interpreted with caution, especially in infants and children, because brain glucose metabolism underlies developmental changes during the first years of life. ${ }^{3}$ This also explains why our PET data were analyzed only visually due to the lack of standardized normal ${ }^{18} \mathrm{~F}-\mathrm{FDG}$ brain controls for semiquantitative assessment in infants and children.

The small sample size of this study and the nonhomogeneity with respect to etiology allowed only descriptive data analysis. The retrospective, cross-sectional nature of this study implies a bias in the selection of our cohort. In addition, outcomes of different pathologies are known to be different (MCD vs ischemic lesions) and thus also may have biased the results. Furthermore, the main parameters we wanted to correlate with surgical outcome-presurgical ${ }^{18}$ F-FDG PET and MRI findings-were already a factor in the presurgical evaluation process, which shaped our cohort.

However, all PET and MRI examinations were performed in a standardized fashion and reevaluated by specialists blinded to the patients' clinical data. Disconnection of the hemispheres is a highly effective but also complex procedure in the treatment of pharmacoresistant epilepsy. Trained and experienced surgeons are needed for excellent outcome. Our small group of patients was operated on by the same neurosurgeon using the vertical perithalamic surgical approach. Nevertheless, to our best knowledge, there is no other study that reported a similar prediction of seizure recurrence by bilateral ${ }^{18} \mathrm{~F}-\mathrm{FDG}$ PET findings or that analyzed presurgical ${ }^{18} \mathrm{~F}-\mathrm{FDG}$ PET in a comparable study design. Nevertheless, our results require confirmation by prospective controlled studies.

\section{Conclusions}

Infants and children with pharmacoresistant epilepsies should undergo a comprehensive examination including
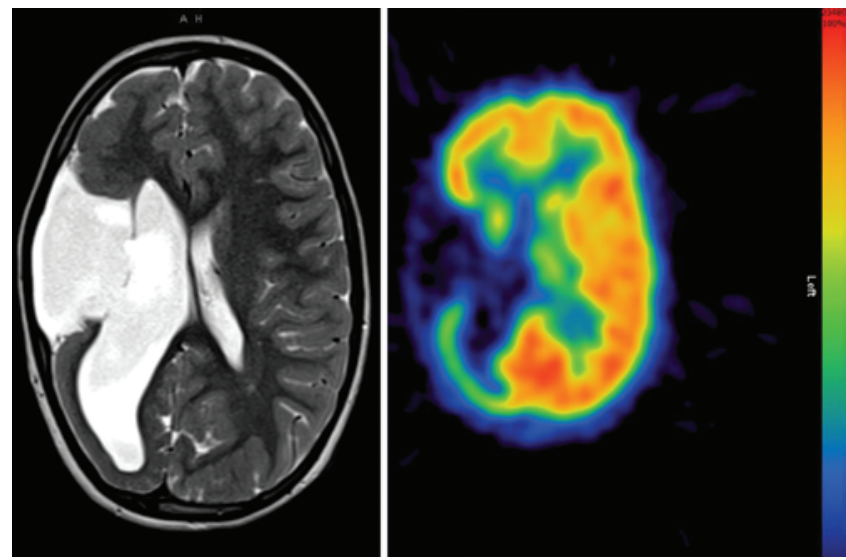

FIG. 2. Case 11. Imaging results from a boy who suffered a perinatal stroke of the right hemisphere as well as polymicrogyria showing bilateral results on MRI, but unilateral results on PET prior to hemispherotomy. Seizure freedom was observed after surgery over a follow-up period of 12 months. The axial T2-weighted sequence (left) shows a large parenchymal defect in the territory of the right middle cerebral artery, occipitolateral thinned cortex, and reduced gray/white matter differentiation, but also polymicrogyria of the right frontal and right occipitomesial cortex, as well as of the occipitomesial cortex of the left hemisphere. The ${ }^{18} \mathrm{~F}-\mathrm{FDG}$ PET axial view (right) presents reduced tracer uptake within the large ischemic defect of the right hemisphere, but not in the regions of polymicrogyria of both hemispheres. Figure is available in color online only.

EEG, seizure semiology, and presurgical imaging with MRI and ${ }^{18} \mathrm{~F}-\mathrm{FDG}$ PET, assessed by an interdisciplinary team prior to hemispherotomy. Functional imaging by ${ }^{18}$ F-FDG PET appears to have both decision-making and predictive value, especially in situations in which clinical information and MRI may not sufficiently support the treatment decision.

\section{References}

1. Blum DE, Ehsan T, Dungan D, Karis JP, Fisher RS: Bilateral temporal hypometabolism in epilepsy. Epilepsia 39:651659, 1998

2. Choi JY, Kim SJ, Hong SB, Seo DW, Hong SC, Kim BT, et al: Extratemporal hypometabolism on FDG PET in temporal lobe epilepsy as a predictor of seizure outcome after temporal lobectomy. Eur J Nucl Med Mol Imaging 30:581-587, 2003

3. Chugani HT, Phelps ME, Mazziotta JC: Positron emission tomography study of human brain functional development. Ann Neurol 22:487-497, 1987

4. Delalande O, Bulteau C, Dellatolas G, Fohlen M, Jalin C, Buret V, et al: Vertical parasagittal hemispherotomy: surgical procedures and clinical long-term outcomes in a population of 83 children. Neurosurgery 60 (2 Suppl 1):ONS19ONS32, 2007

5. Dorfer C, Czech T, Dressler A, Gröppel G, MühlebnerFahrngruber A, Novak K, et al: Vertical perithalamic hemispherotomy: a single-center experience in 40 pediatric patients with epilepsy. Epilepsia 54:1905-1912, 2013

6. González-Martínez JA, Gupta A, Kotagal P, Lachhwani D, Wyllie E, Lüders HO, et al: Hemispherectomy for catastrophic epilepsy in infants. Epilepsia 46:1518-1525, 2005

7. Griessenauer CJ, Salam S, Hendrix P, Patel DM, Tubbs RS, Blount JP, et al: Hemispherectomy for treatment of refractory epilepsy in the pediatric age group: a systematic review. J Neurosurg Pediatr 15:34-44, 2015 
8. Gröppel G, Dorfer C, Mühlebner-Fahrngruber A, Dressler A, Porsche B, Czech T, et al: Improvement of language development after successful hemispherotomy. Seizure 30:70-75, 2015

9. Hallbook T, Ruggieri P, Adina C, Lachhwani DK, Gupta A, Kotagal P, et al: Contralateral MRI abnormalities in candidates for hemispherectomy for refractory epilepsy. Epilepsia 51:556-563, 2010

10. Hamacher K, Coenen HH, Stöcklin G: Efficient stereospecific synthesis of no-carrier-added 2-[ $\left.{ }^{18} \mathrm{~F}\right]$-fluoro-2-deoxy-Dglucose using aminopolyether supported nucleophilic substitution. J Nucl Med 27:235-238, 1986

11. Kossoff EH, Vining EPG, Pillas DJ, Pyzik PL, Avellino AM, Carson BS, et al: Hemispherectomy for intractable unihemispheric epilepsy etiology vs outcome. Neurology 61:887890, 2003

12. Kumar A, Chugani HT: The role of radionuclide imaging in epilepsy, part 1: sporadic temporal and extratemporal lobe epilepsy. J Nucl Med 54:1775-1781, 2013

13. Kumar A, Chugani HT: The role of radionuclide imaging in epilepsy, part 2: epilepsy syndromes. J Nucl Med 54:19241930, 2013

14. Kwan P, Arzimanoglou A, Berg AT, Brodie MJ, Allen Hauser W, Mathern G, et al: Definition of drug resistant epilepsy: consensus proposal by the ad hoc Task Force of the ILAE Commission on Therapeutic Strategies. Epilepsia 51:10691077,2010

15. Moosa ANV, Gupta A, Jehi L, Marashly A, Cosmo G, Lachhwani D, et al: Longitudinal seizure outcome and prognostic predictors after hemispherectomy in 170 children. Neurology 80:253-260, 2013

16. Moosa ANV, Jehi L, Marashly A, Cosmo G, Lachhwani D, Wyllie E, et al: Long-term functional outcomes and their predictors after hemispherectomy in 115 children. Epilepsia 54:1771-1779, 2013

17. Ramey WL, Martirosyan NL, Lieu CM, Hasham HA, Lemole GM Jr, Weinand ME: Current management and surgical outcomes of medically intractable epilepsy. Clin Neurol Neurosurg 115:2411-2418, 2013

18. Rasmussen T: Hemispherectomy for seizures revisited. Can J Neurol Sci 10:71-78, 1983

19. Rintahaka PJ, Chugani HT, Messa C, Phelps ME: Hemimegalencephaly: evaluation with positron emission tomography. Pediatr Neurol 9:21-28, 1993

20. Rosenow F, Lüders H: Presurgical evaluation of epilepsy. Brain 124:1683-1700, 2001

21. Uijl SG, Leijten FSS, Arends JBAM, Parra J, van Huffelen
AC, Moons KGM: Prognosis after temporal lobe epilepsy surgery: the value of combining predictors. Epilepsia 49:1317-1323, 2008

22. Wieser HG, Blume WT, Fish D, Goldensohn E, Hufnagel A, King D, et al: ILAE Commission Report. Proposal for a new classification of outcome with respect to epileptic seizures following epilepsy surgery. Epilepsia 42:282-286, 2001

23. Willmann O, Wennberg R, May T, Woermann FG, Pohlmann-Eden B: The contribution of 18F-FDG PET in preoperative epilepsy surgery evaluation for patients with temporal lobe epilepsy A meta-analysis. Seizure 16:509-520, 2007

24. Wirrell EC: Predicting pharmacoresistance in pediatric epilepsy. Epilepsia 54 (2 Suppl 2):19-22, 2013

25. Varrone A, Asenbaum S, Vander Borght T, Booij J, Nobili F, Någren K, et al: EANM procedure guidelines for PET brain imaging using $\left[{ }^{18} \mathrm{~F}\right] \mathrm{FDG}$, version 2 . Eur J Nucl Med Mol Imaging 36:2103-2110, 2009

26. Vinton AB, Carne R, Hicks RJ, Desmond PM, Kilpatrick C, Kaye AH, et al: The extent of resection of FDG-PET hypometabolism relates to outcome of temporal lobectomy. Brain 130:548-560, 2007

27. Zhang J, Liu W, Chen H, Xia H, Zhou Z, Mei S, et al: Multimodal neuroimaging in presurgical evaluation of drug-resistant epilepsy. Neuroimage Clin 4:35-44, 2013

\section{Disclosures}

The authors report no conflict of interest concerning the materials or methods used in this study or the findings specified in this paper.

\section{Author Contributions}

Conception and design: Feucht, Traub-Weidinger. Acquisition of data: Weidinger, Gröppel. Analysis and interpretation of data: Feucht, Traub-Weidinger, Karanikas, Kasprian. Drafting the article: Feucht, Traub-Weidinger, Weidinger. Critically revising the article: all authors. Reviewed submitted version of manuscript: all authors. Approved the final version of the manuscript on behalf of all authors: Feucht. Statistical analysis: Traub-Weidinger, Weidinger. Administrative/technical/material support: Gröppel, Wadsak, Dressler, Mühlebner. Study supervision: Feucht.

\section{Correspondence}

Martha Feucht, Department of Paediatrics, Medical University of Vienna, Währinger Gürtel 18-20A-1090 Wien, Austria. email: martha.feucht@meduniwien.ac.at. 